\title{
Vocal Impact of a Prolonged Reading Task in Dysphonic versus Normophonic Female Teachers
}

\section{Angélique REMACLE, Dominique MORSOMME, Elise BERRUE, Camille FINCK}

Unité de Logopédie de la voix

Faculté de Psychologie et des Sciences de l'Education

Université de Liège

Belgium 


\section{Background}

- PhD about vocal loading

- Previous study ${ }^{1}$ : vocal impact of a 2-hour reading task in normophonic females without professional voice use

- This study2: vocal impact of a 2-hour reading task in normophonic and dysphonic female teachers

(1) Remacle A, Finck C, Roche A, Morsomme D. Vocal Impact of a Prolonged Reading Task at Two Intensity Levels: Objective Measurements and Subjective Self-Ratings. J. Voice. In press (2) Remacle A, Morsomme D, Berrué E, Finck C. Vocal Impact of a Prolonged Reading Task in Dysphonic versus Normophonic Female Teachers. J. Voice. In Press 


\section{Methods: participants}
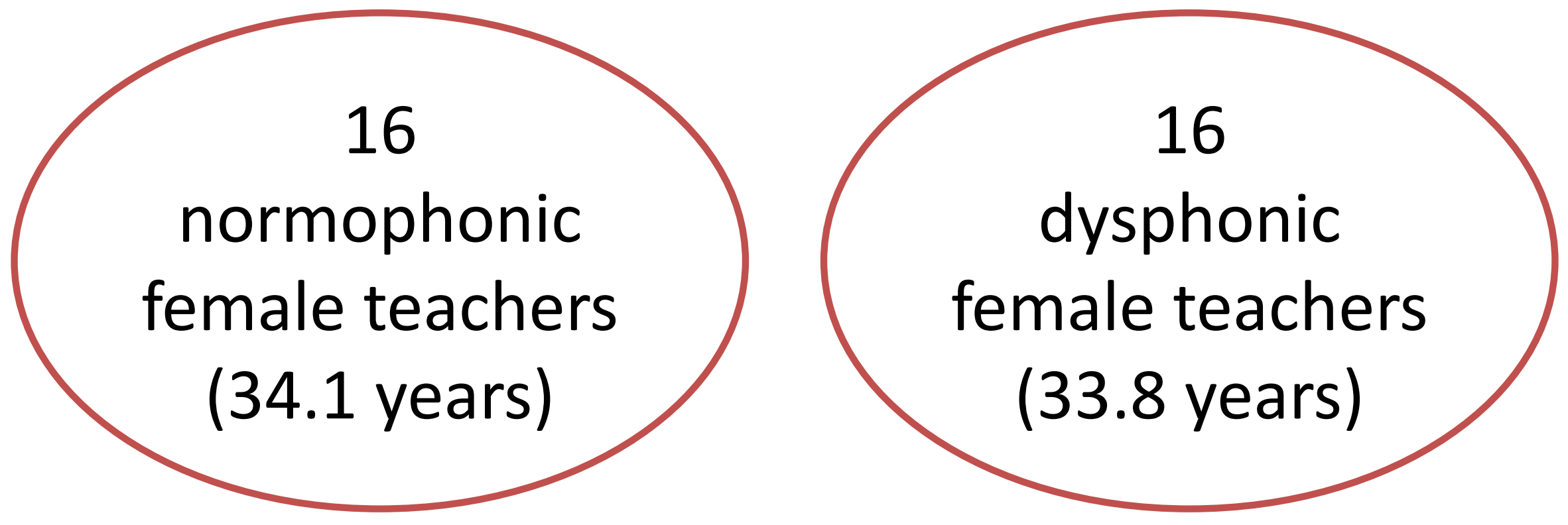


\section{Methods: loading task}

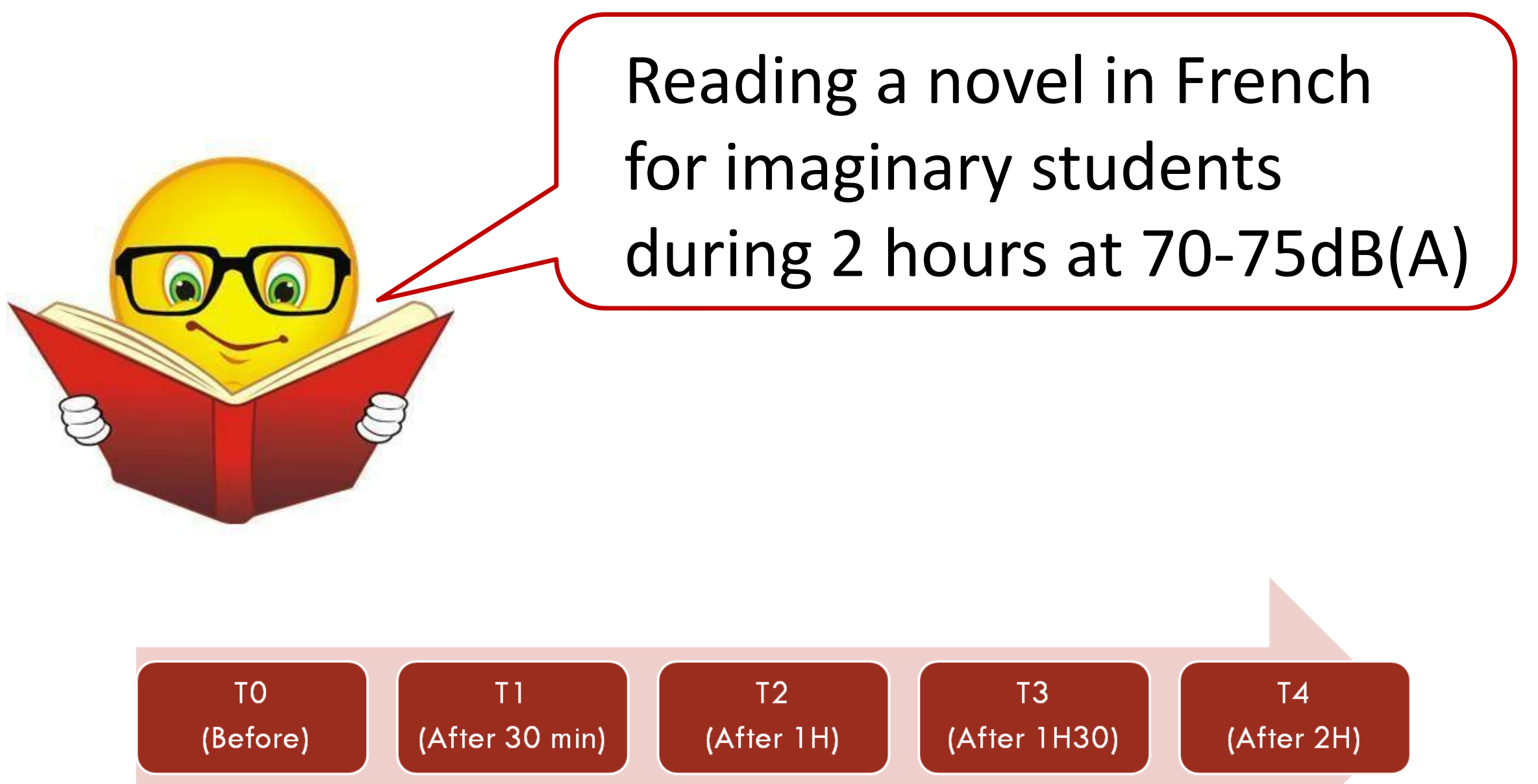




\section{Methods: evaluation protocol}

Before the task and every 30 minutes

- Acoustic analysis (MDVP)

F0, Jitter\%, Shimmer\%

- Voice range measurements (VRP)

Frequency and intensity

- Aerodynamic measurements (Aerophone II)

Maximum Phonation Time

Subglottic Pressure, SPL 


\section{The questions are:}

- What are the effects of a two-hour reading task on teachers' voice?

- Does the vocal load affect differently the dysphonic teachers than the healthy teachers?

Statistical analysis: repeated measures ANOVA

$$
* p<.05
$$




\section{Results: acoustic measurements}

F0 *

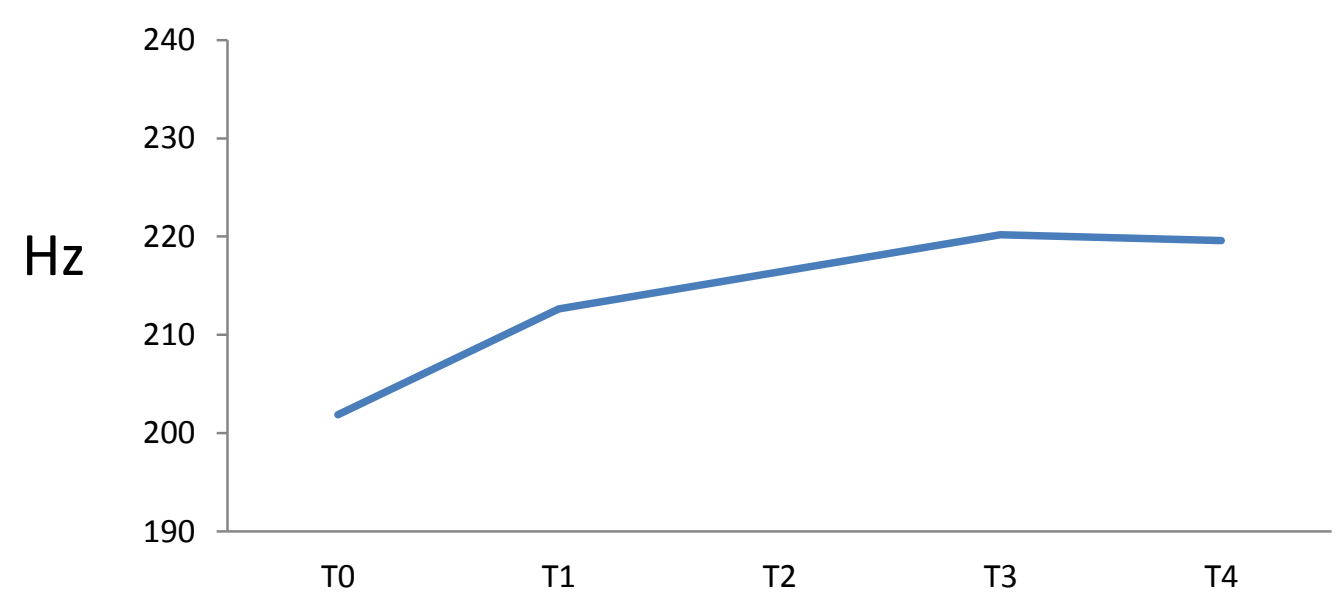




\section{Results: acoustic measurements}

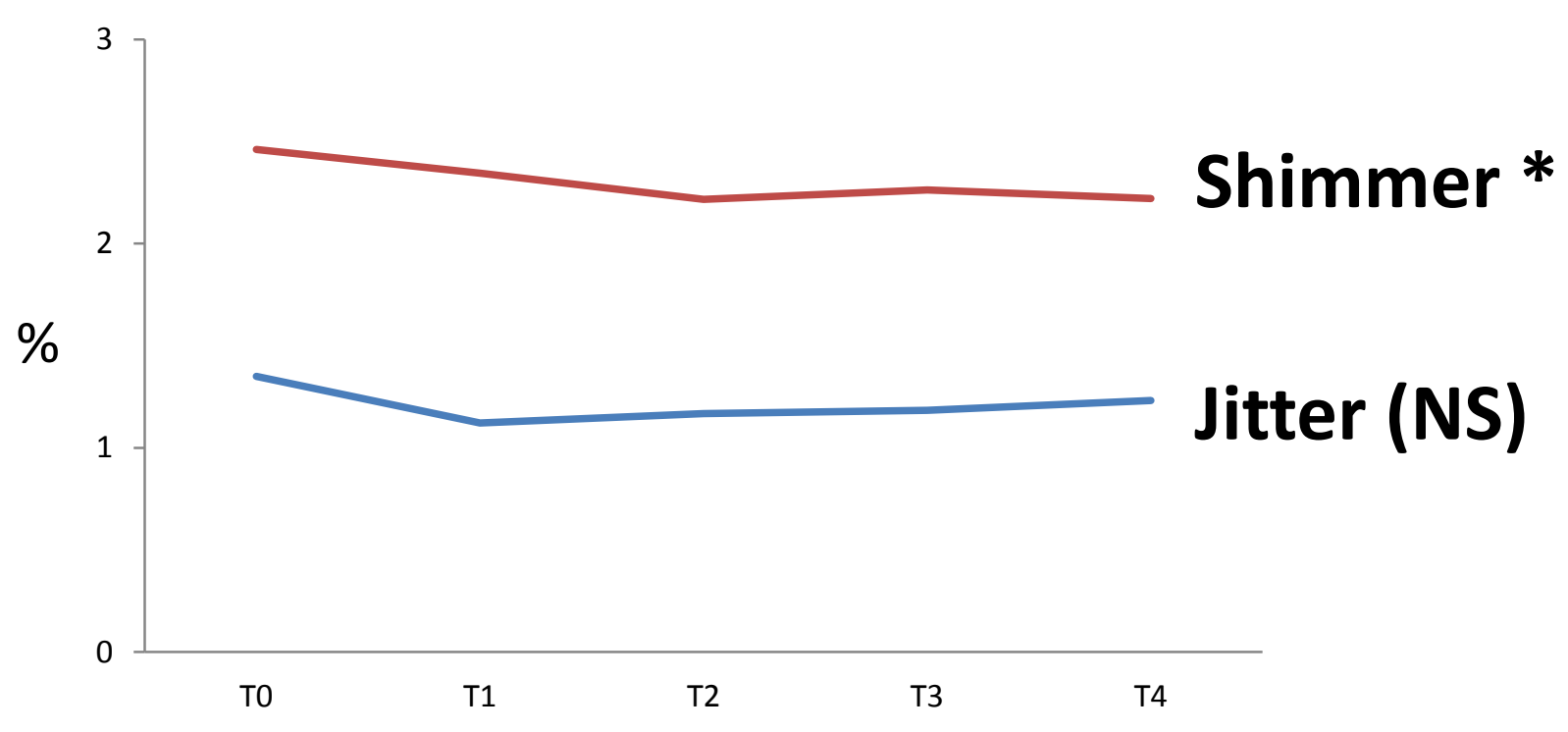




\section{Results: voice range measurements}
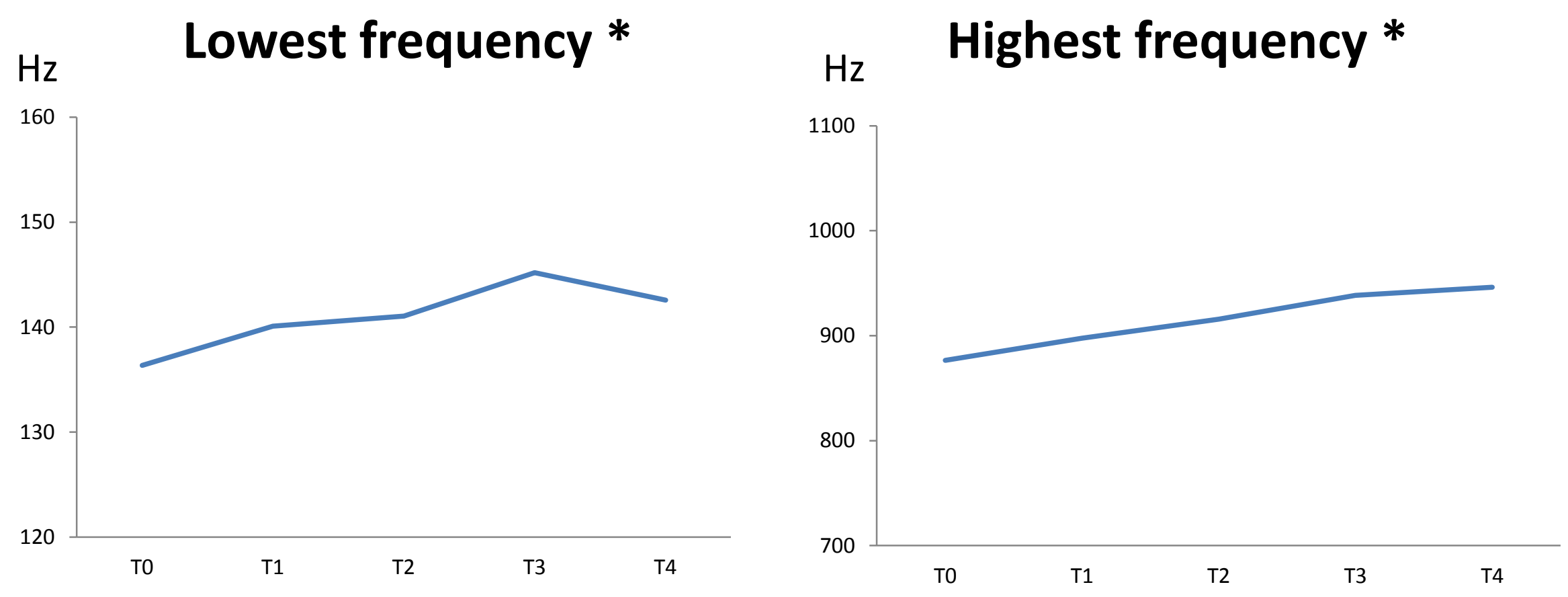

Frequency range: no significant effect of duration 


\section{Results: voice range measurements}
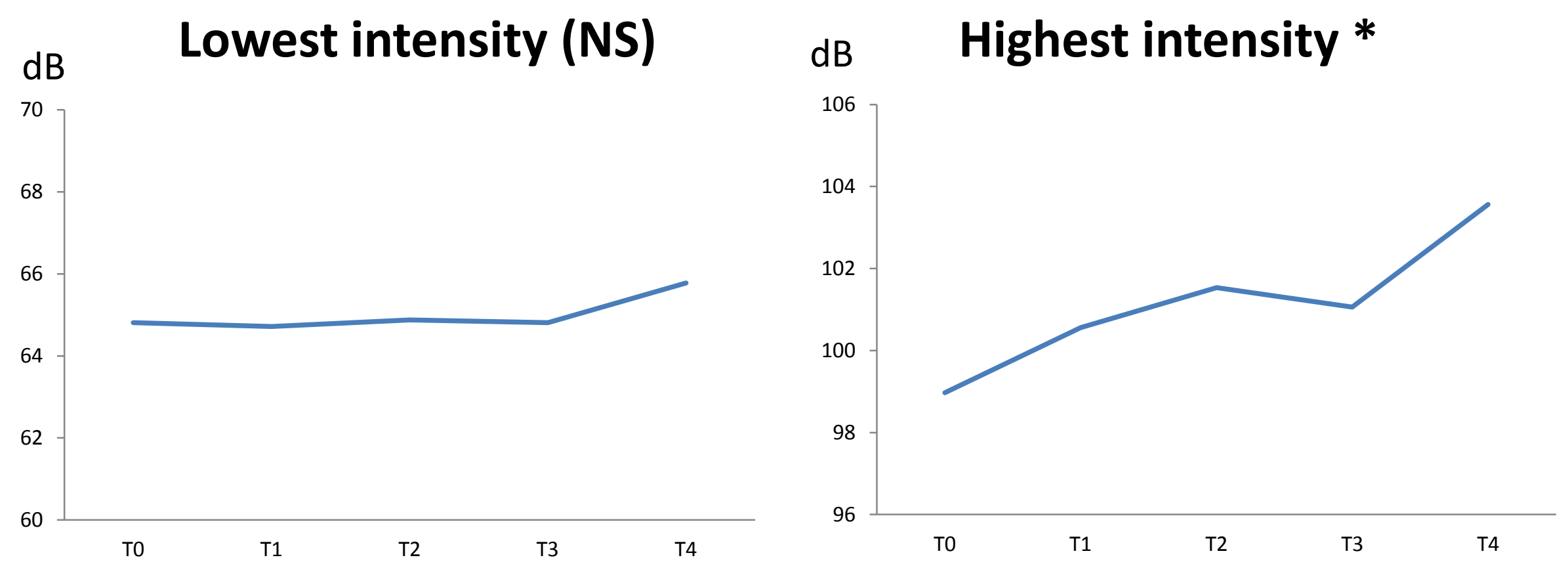

Intensity range significantly increases during the reading 


\section{Results: aerodynamic measurements}

Maximum Phonation Time

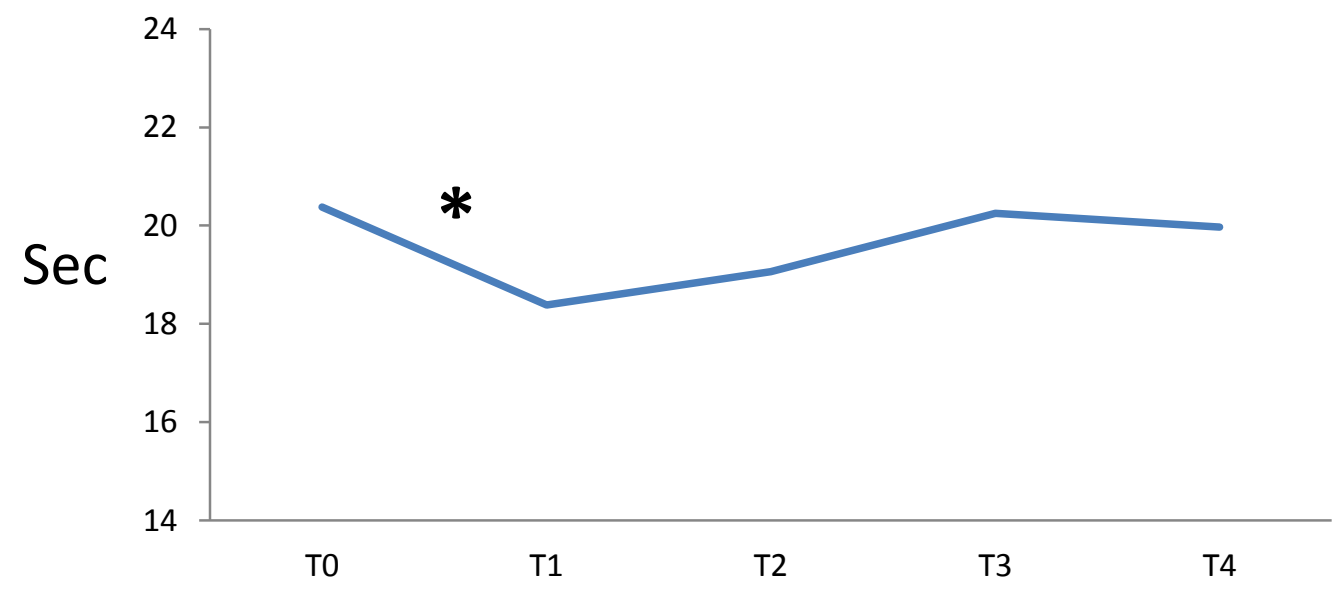




\section{Results: aerodynamic measurements}

Subglottic Pressure

$\mathrm{cmH}_{2} \mathrm{O}$

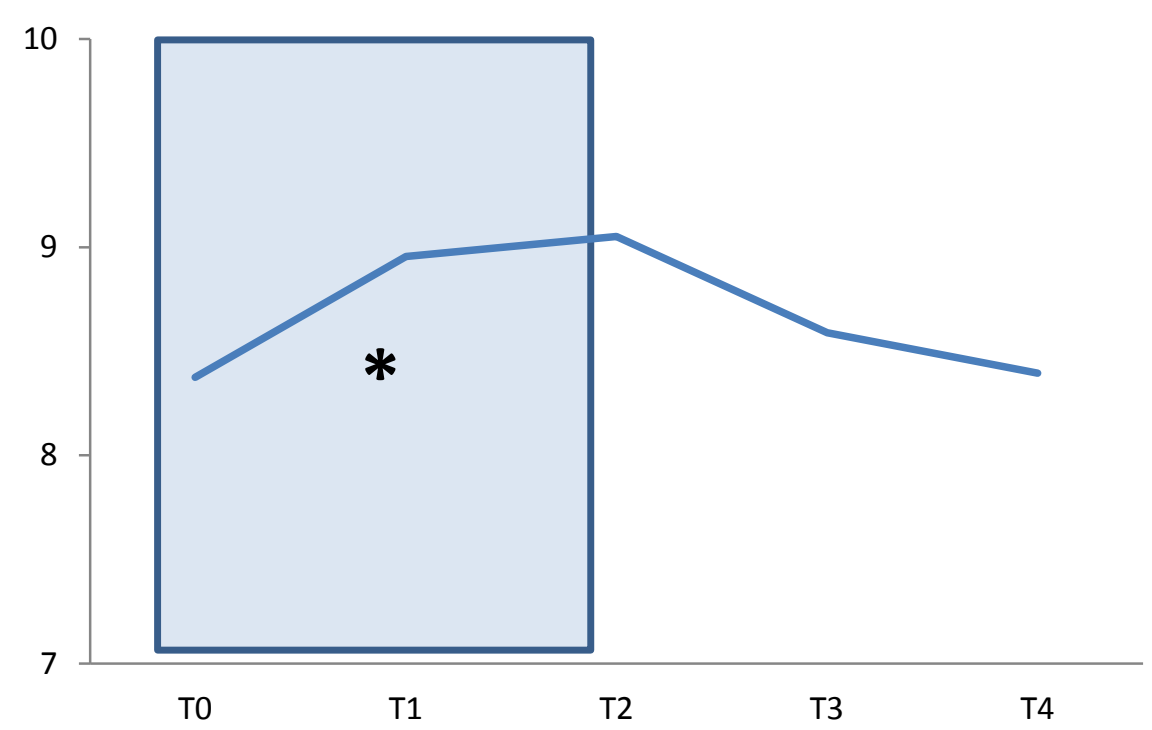

SPL

$d B$

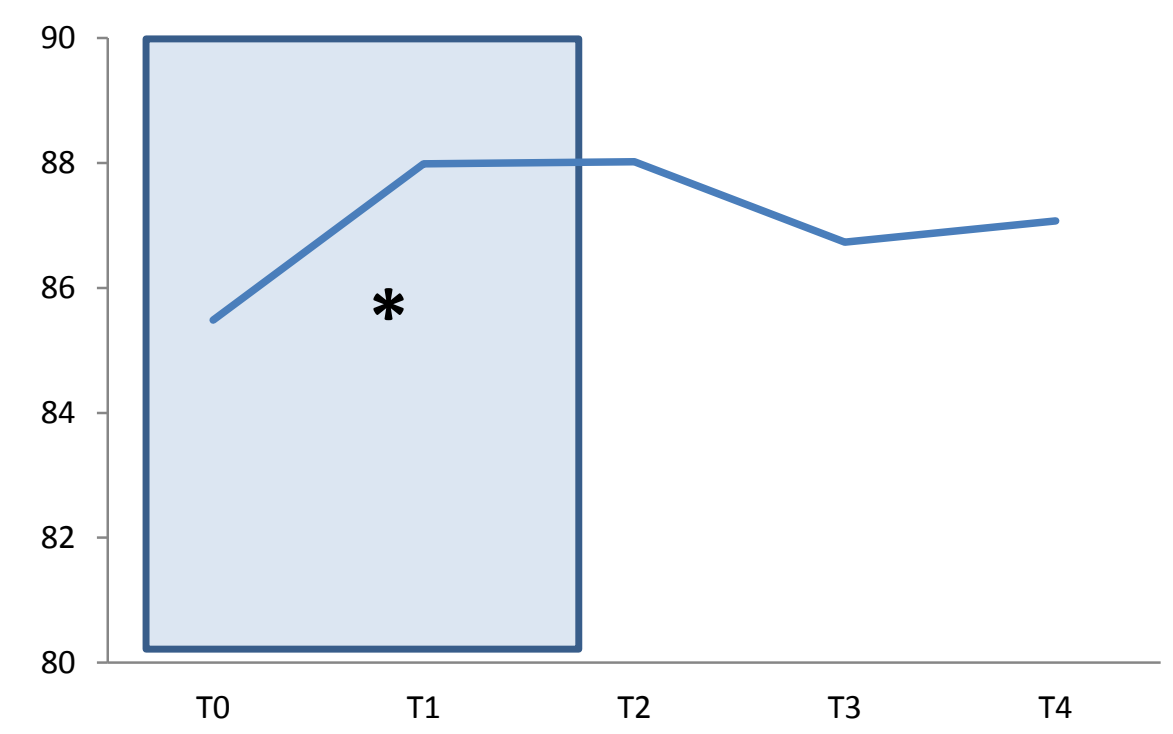




\section{Results: aerodynamic measurements}

Subglottic Pressure

$\mathrm{cmH}_{2} \mathrm{O}$

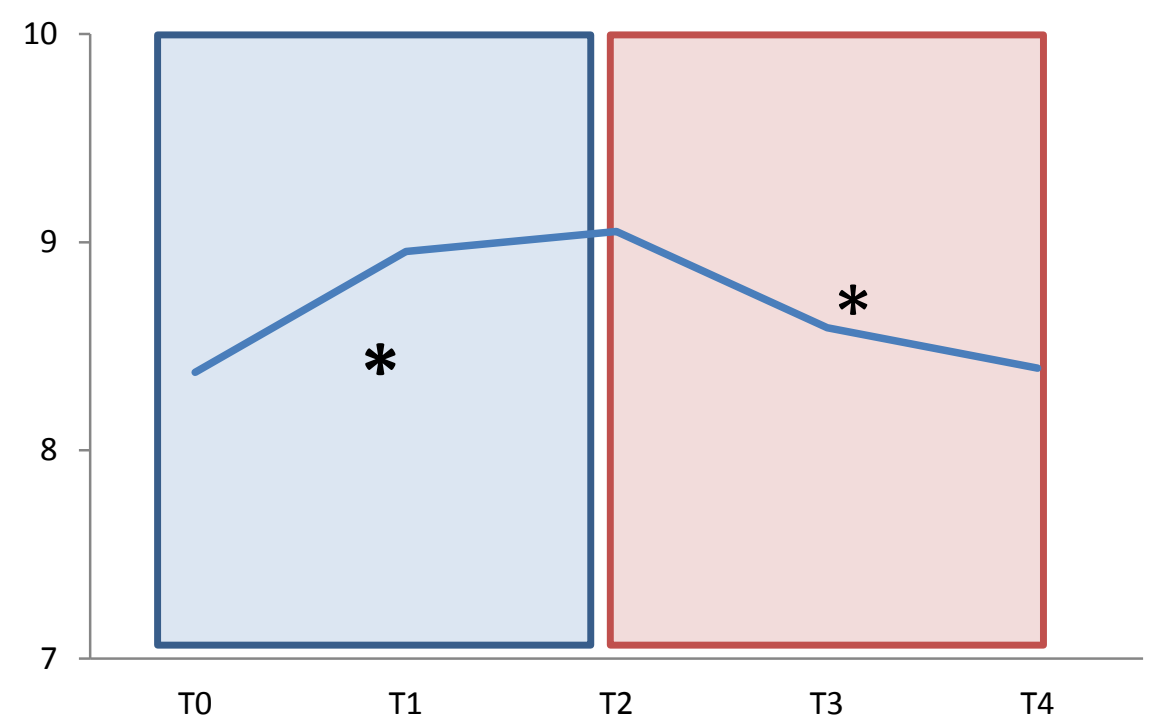

SPL

$d B$

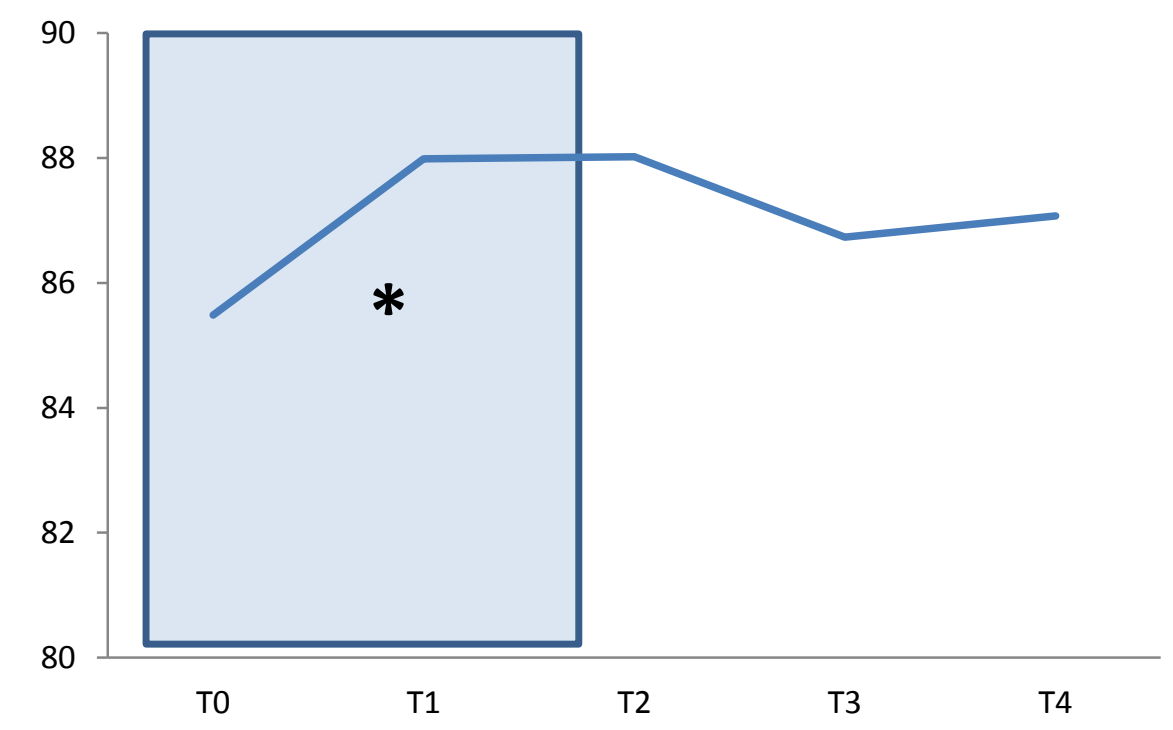




\section{Results: few differences between normophonic and dysphonic groups}

Highest frequency*

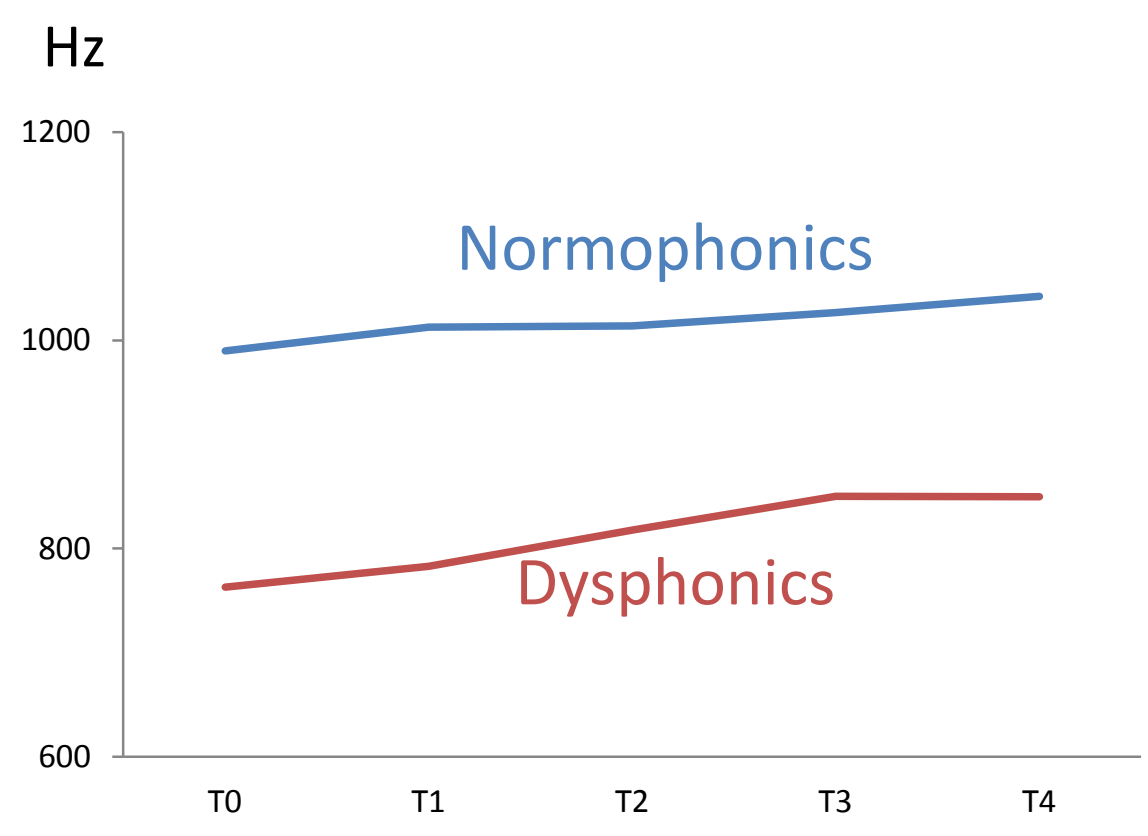

Frequency range*

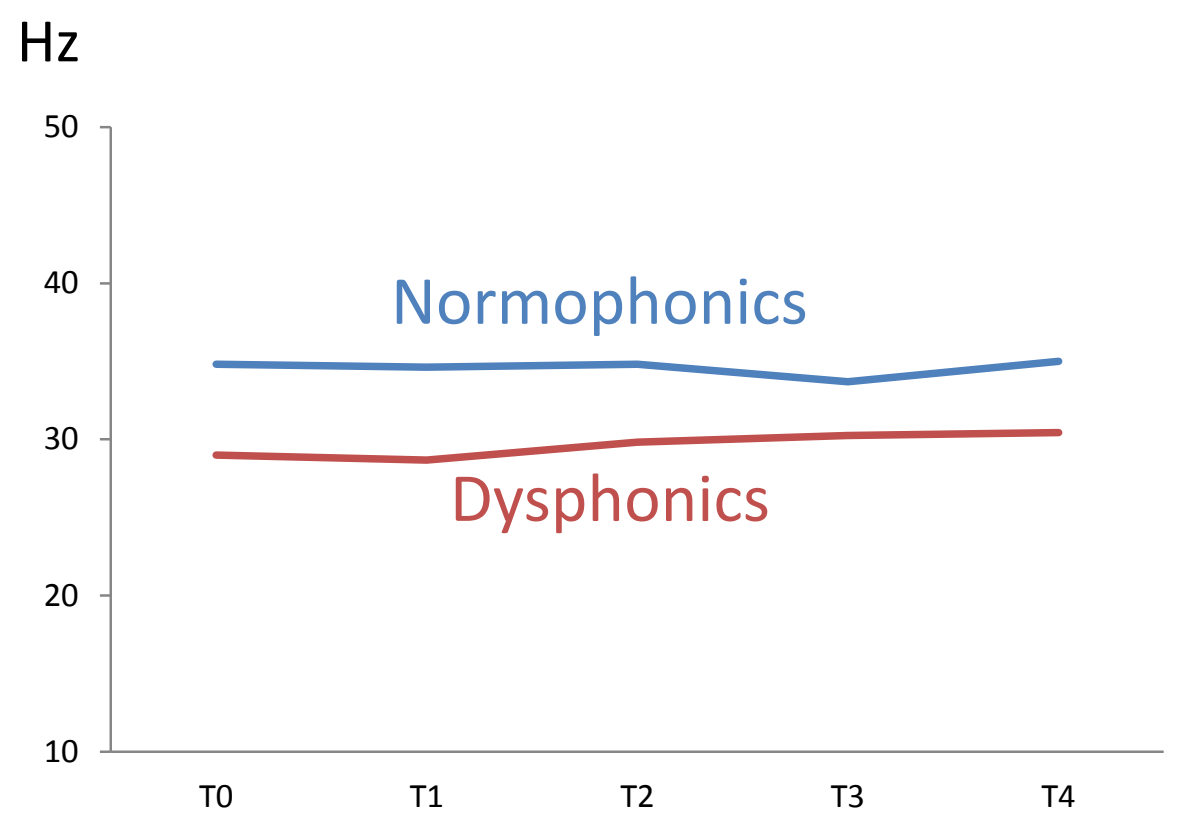




\section{Results: few differences between normophonic and dysphonic groups}

Subglottic Pressure*

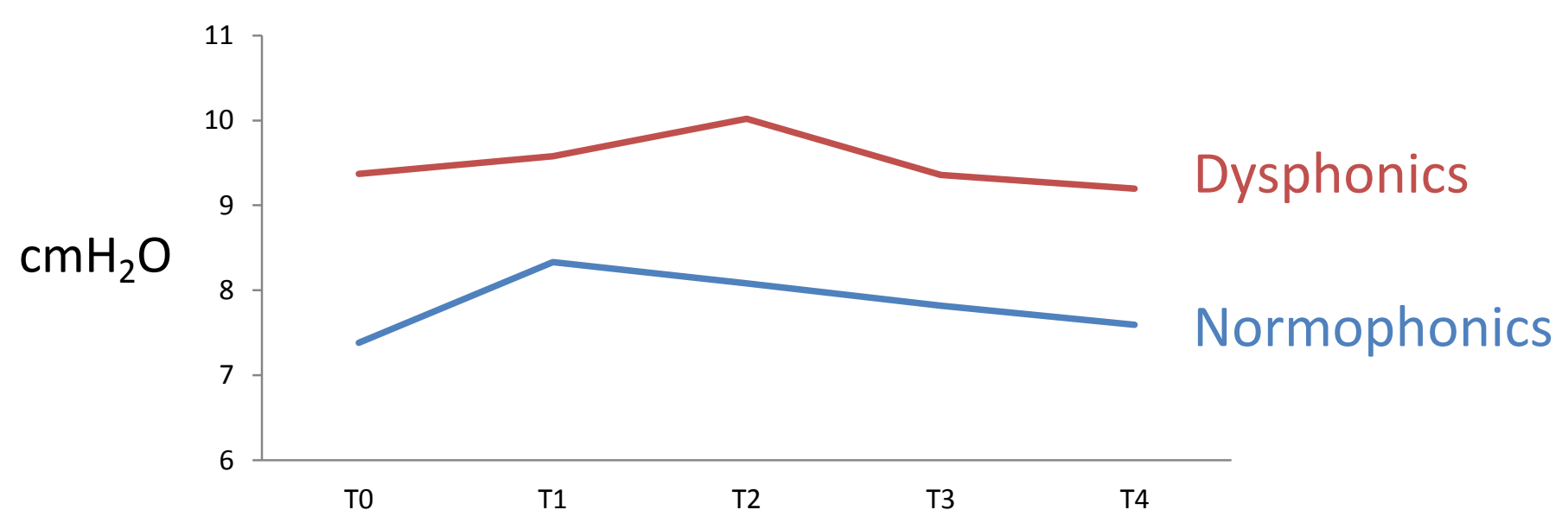




\section{Results: interaction between the duration and the group only for}

Lowest Frequency*

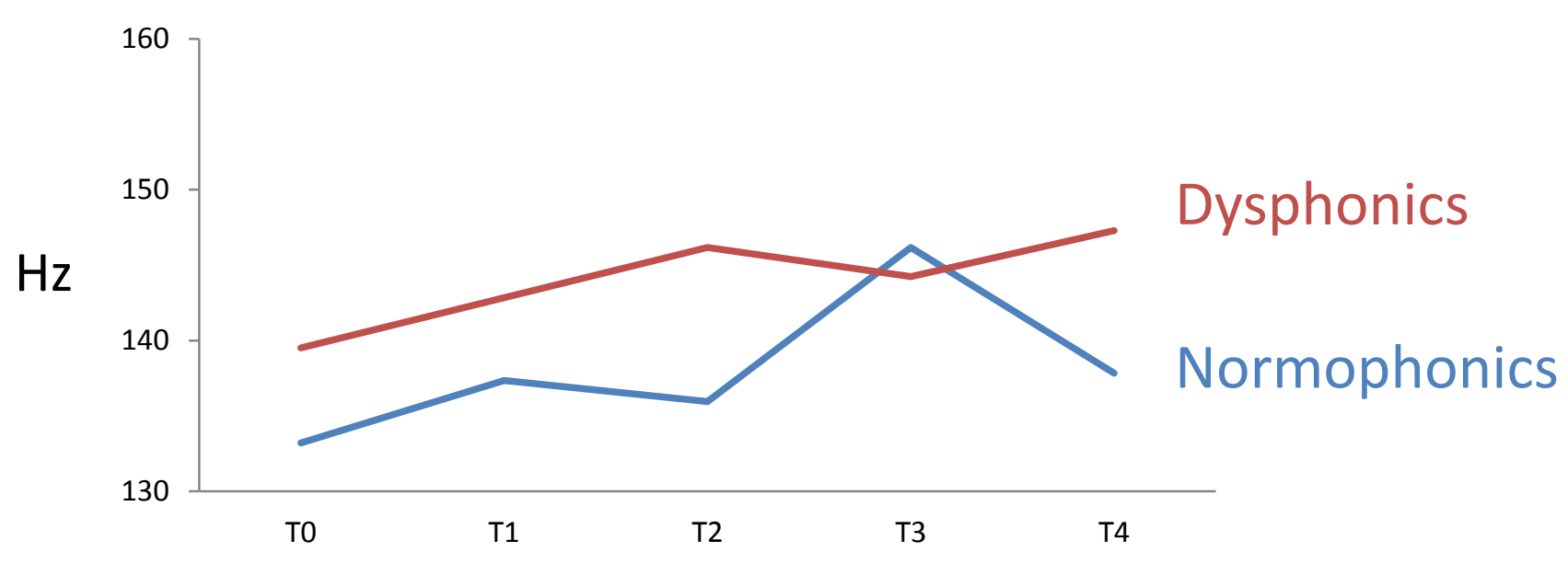




\section{Conclusions: \\ effects of a 2-hour reading task}

- Acoustic analysis and voice range measurements suggest an

increased laryngeal tension

- Aerodynamic measurements suggest

1) lower voice efficiency - increased

viscosity and stiffness of the vocal folds

2) adaptation to vocal loading 


\section{Conclusions:}

\section{normophonic versus dysphonic groups}

- Few differences between both groups

- Voice evolution through the reading task similar for the normophonic and the dysphonic groups 
Unité de Logopédie de la voix

Faculté de Psychologie et des Sciences de l'Education

Université de Liège

Belgium

Angelique.Remacle@ulg.ac.be

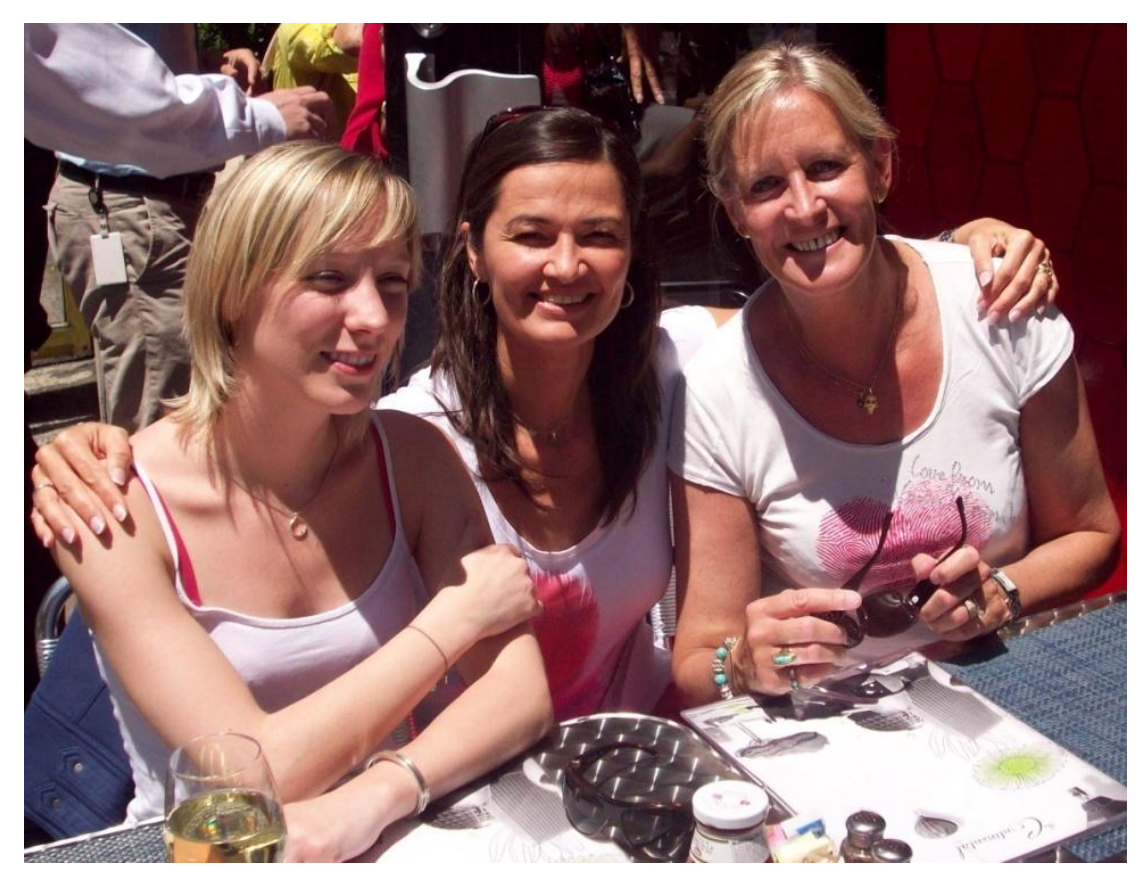

Thank You! 